\title{
THE BIRD BURIAL AND ATHLETE'S PARADE
}

And I buried him,

his lungs, the tiny bellows

that rotted and stove in.

Under the leaves

the vest of bones lay open.

Now in the park

the racers, the strutters come forward

for their medals.

I give them your wings

cooking in their soup,

the tarted feet and belly feathers

to the runners.

I take the spine

so it might be a beam for my sail,

turning idly tonight.

Let us drift sparrow. 